\title{
The Absorption Line Systems of the Supershell LMC2 in the Large Magellanic Cloud
}

\author{
Adeline Caulet
}

Astronomy Department, University of Illinois, Urbana, IL 61801, USA

\begin{abstract}
The interstellar medium of LMC2, a well studied supershell in the Large Magellanic Cloud, has been probed in UV and optical absorption lines. The data allow to derive the kinematics, abundances and depletions of gas clouds in this supershell. The relative gas-phase abundances of observed elements with respect to sulphur are useful to determine the origins of the supershell absorption-line clouds.
\end{abstract}

\section{Introduction}

Supershells (or supergiant shells, SGS) which are large-scale structures in galaxies have a multi-phase ISM. They are made of gas clouds with a large range of velocities, densities and temperatures. It is interesting to determine whether these clouds originate in a cold or warm disk or in a hot halo, so that we may understand the role of supershells in galaxies. Because supershells represent large reservoirs of energy, and can reach large dimensions in and above galactic disks, they can play an important role in galactic structure, e.g., in the formation and evolution of galactic halos, via the interaction of multiple supernovae explosions with the ISM.

\section{Observations}

To probe the multi-phase ISM of the well-studied supershell LMC2 in the Large Magellanic Cloud, we have analyzed the high resolution optical (Caulet \& Newell 1996) and UV (Fig. 1) spectra of 7 OB supergiant stars in the field of LMC2. Line-profile fits of the absorption lines give the velocities, column densities, and Doppler widths of the absorbing clouds along the stellar lines-of-sight. For the spectral ranges observed, the ions C I $(\lambda 1260, \lambda 1560)$, and Ti II $(\lambda 3384)$ arise in cold and warm neutral clouds, and provide information about cool clouds kinematically associated with HI structures (such as the flocculent HI seen in ATCA maps by Kim et al. 1998), the compressed gas behind the outward moving shocks, and cool clouds embedded in the hot gas inside the SGS. The ionized medium comes from both photoionization of the SGSs and post-shock relaxation of the gas: Mg II $(\lambda 1240)$, S II $(\lambda 1250, \lambda 1253, \lambda 1259)$, Si II $(\lambda 1260)$, and Ca II ( $\lambda 3934)$. The photo-ionized gas and coronal gas are traced by the C IV $\lambda \lambda 1548$, $1550\left(\mathrm{~T} \sim 10^{5} \mathrm{~K}\right)$ lines. $\mathrm{N} \mathrm{V} \lambda \lambda 1238,1240\left(\mathrm{~T} \sim 2 \times 10^{5} \mathrm{~K}\right)$ lines trace shocks, and collisionally excited gas as $\mathrm{N}^{+4}$ may arise at the interfaces of cloud-hot gas in the SGS cavities. Many cold, warm and hot clouds are detected over the 
whole velocity range between the Galactic velocities $\left(\mathrm{v} \leq 50 \mathrm{~km}^{-1}\right)$ and the LMC $\left(200-360 \mathrm{~km}^{-1}\right)$ velocities. While C IV is generally observed, no N V is detected down to a $3 \sigma$ detection limit of $10 \mathrm{~m} \AA$. The ions $\mathrm{Si} \mathrm{II}^{*}(\lambda 1264), \mathrm{C}$ I $(\lambda 1260)$, $\mathrm{Mg}$ II $(\lambda 1240)$ are identified in some clouds.
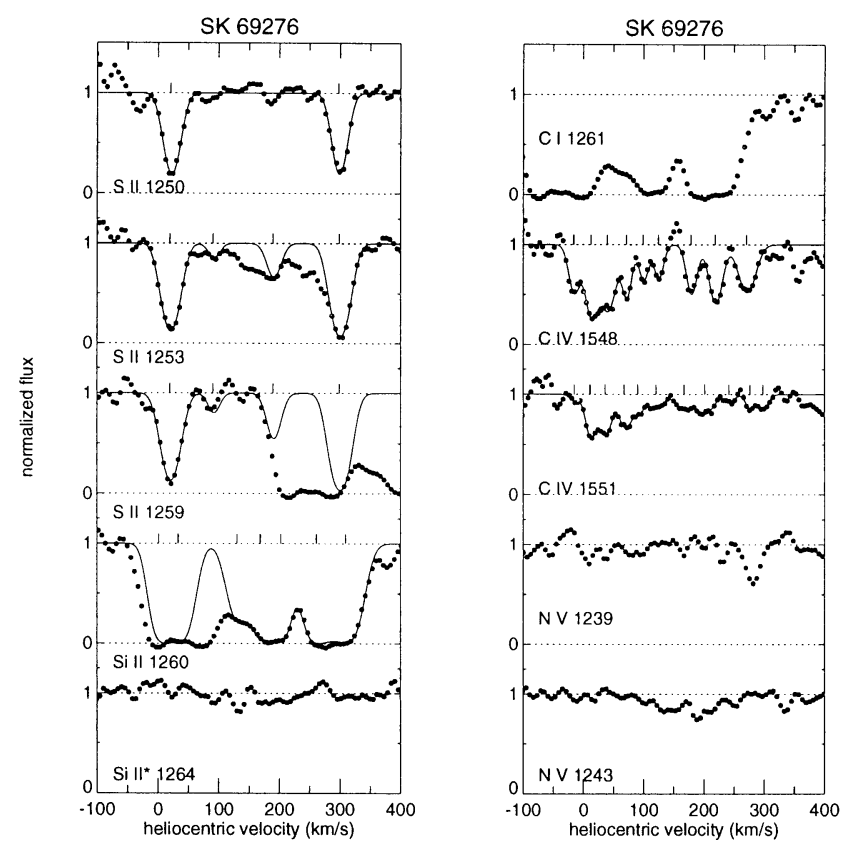

Figure 1. UV absorption lines toward SK-69276: no C I, Si II*, N V

Because it is not possible to determine directly distances for these clouds within the LMC, we examined first their relative positions by comparing their velocities with those of structures observed in emission lines, and neutral gas, and in absorption lines for other published lines-of-sight in the vicinity of LMC2 and the 30 Doradus region (see Paper I). Then, for each cloud, we plotted the gas-phase abundances of elements (relative to the "undepleted" sulpher) against their condensation temperatures to determine if these clouds are representative of disk or halo clouds (see Savage \& Sembach 1996; Welty et al. 1997). The completed study will be reported in a refereed paper.

Acknowledgments. The author thanks You-Hua Chu and the Astronomy Deparment of the University of Illinois for their hospitality during her visit and the financial support to attend this symposium.

\section{References}

Caulet, A., \& Newell, R. 1996, ApJ, 465, 205

Kim, S., et al. 1998, ApJ, 503, 674

Savage, B.D., \& Sembach, K.R. 1996, ARA\&A, 34, 279

Welty, D.E., et al. 1997, ApJ, 489, 672 